\title{
Neuroendocrine cell proliferations in lungs explanted for fibrotic interstitial lung disease and emphysema
}

\author{
Susan Shyu ${ }^{1,2}$, Jonathon E. Heath ${ }^{1,2}$, Allen P. Burke ${ }^{1,2}$ \\ ${ }^{1}$ Department of Pathology, University of Maryland Medical System, Baltimore, MD, \\ United States; ${ }^{2}$ Department of Pathology, University of Maryland School of Medicine, \\ Baltimore, MD, United States
}

\begin{abstract}
Summary
Using recently proposed pathological criteria, we determined the incidence of neuroendocrine cell proliferation in a series of explants with lung disease. Cases were defined as $\mathrm{NECH} \mathrm{(} \geq 3$ bronchioles with $\geq 5$ endocrine cells), borderline diffuse neuroendocrine cell hyperplasia (DPNECH) (1-3 tumourlets with or without $\mathrm{NECH}$ ), and DPNECH ( $\geq 3$ tumourlets with $\mathrm{NECH}$ ). Endocrine cells were identified by immunohistochemical staining for synaptophysin. There were 65 explants with interstitial lung disease (57 with non-sarcoid fibrotic lung disease, 8 with sarcoidosis), and 21 with centrilobular emphysema. Over one-third of all explant cases demonstrated histological criteria for $\mathrm{NECH}$. There were three cases of $\mathrm{DPNECH}$ in the non-sarcoid fibrotic lung disease group (5\%) and 20 cases of $\mathrm{NECH}(35 \%)$. The emphysema group had one case of DPNECH (5\%), two cases of borderline DPNECH $(10 \%)$, and seven cases with $\mathrm{NECH}(33 \%)$. The sarcoidosis group had two cases of DPNECH (25\%) and three cases of $\mathrm{NECH}(38 \%)$. $\mathrm{NECH}$ is common in interstitial lung disease and emphysema. These results suggest that fibrotic lung disease is a predisposing factor for neuroendocrine cell proliferation, in addition to the known risk of epithelial neoplasms.
\end{abstract}

Key words: Neuroendocrine cell hyperplasia; NECH; fibrotic lung disease; sarcoidosis; lung transplantation.

Received 6 April, revised 9 August, accepted 17 August 2018

Available online 22 October 2018

\section{INTRODUCTION}

Neuroendocrine cell proliferations of the lung range from neuroendocrine cell hyperplasia $(\mathrm{NECH})$ to malignant neoplasms, namely carcinoids, small cell carcinoma, and large cell carcinoma. The mechanism for the development of welldifferentiated lung neuroendocrine tumours-low-grade (typical carcinoid) and intermediate-grade (atypical) carcinoids - is still not well understood. While the majority of cases arise de novo, a percentage develop in patients with diffuse idiopathic neuroendocrine cell hyperplasia (DIPNECH), a recently classified preneoplastic lesion in the 2015 World Health Organization (WHO) classification of lung tumours. ${ }^{1,2}$

Standardised clinical or pathological criteria for the diagnosis of DIPNECH and non-idiopathic neuroendocrine cell hyperplasia (NECH) have yet to be established. WHO defines DIPNECH as 'a generalized proliferation of scattered single cells, small nodules (neuroendocrine bodies) or linear proliferations of pulmonary neuroendocrine cells (PNCs) that may be confined to the bronchial and bronchiolar epithelium, include local extraluminal proliferation in the form of tumorlets, or extend to the development of carcinoid tumors'. ${ }^{2}$ However, no quantitative criteria, such as the minimum number of PNCs to indicate a 'proliferation' or the number of involved airways for a 'generalised' process, are provided. It is also unclear under what circumstances the presence of NECH should be classified as idiopathic versus secondary.

This can pose a diagnostic problem for pathologists as NECH and carcinoid tumourlets are not uncommon in lung surgical specimens. ${ }^{3,4} \mathrm{NECH}$ has primarily been described as case reports in association with emphysema and idiopathic pulmonary fibrosis. ${ }^{5,6}$ Most studies have emphasised the presence of NECH in patients with carcinoid tumours and have found symptoms of DIPNECH to be rare, although $10 \%$ have multiple tumourlets. ${ }^{7}$ The incidence of $\mathrm{NECH}$ in emphysema or idiopathic pulmonary fibrosis is unknown.

Definitions for NECH and DIPNECH have recently been proposed by Marchevsky et al. ${ }^{8}$ Using the proposed definitions, we sought to determine the incidence of neuroendocrine cell proliferations in chronic lung disease requiring transplantation.

\section{MATERIALS AND METHODS}

We performed a retrospective review on consecutive lung specimens explanted for fibrotic lung disease and emphysema between December 2013 and June 2016. Haematoxylin and eosin slides of each case were reviewed by a pathologist, and two representative blocks with the greatest potential for pulmonary neuroendocrine cell proliferations were selected for synaptophysin staining. Immunohistochemical detection of synaptophysin (27G12) was performed on $4 \mu \mathrm{m}$ sections of formalin fixed, paraffin embedded (FFPE) tissue mouse monoclonal predilute antibody from Leica (Germany). Staining was done on the Leica Bond III automated immunohistochemistry slide stainer using online heat induced epitope retrieval method in ER2 buffer (Leica) for $20 \mathrm{~min}$ at $\mathrm{pH}$ 9.0. The staining was visualised using the Bond Polymer Refine Detection kit (Leica). The slides were subsequently counterstained with haematoxylin. Appropriate positive and negative controls were included in each staining run.

Cases were defined as proposed by Marchevsky et al. as NECH $(\geq 3$ bronchioles with $\geq 5$ endocrine cells), borderline diffuse neuroendocrine cell hyperplasia (DPNECH) (1-3 tumourlets without NECH or 1-2 tumourlets with NECH), and DPNECH ( $\geq 3$ tumourlets with NECH). The synaptophysin stained slides were reviewed by two pathologists, and consensus was 
achieved in all cases. We also looked for obliterative bronchiolitis, described by Aguayo as neuroendocrine cell clusters obliterating the walls of terminal bronchioles and protruding into the lumen of associated air spaces. ${ }^{9}$

\section{RESULTS}

There was a total of 86 explants, 57 with non-sarcoid fibrotic lung disease, eight with sarcoidosis, and 21 with centrilobular emphysema. The majority (54/57) of non-sarcoid fibrotic lung disease specimens were explants for usual interstitial pneumonia (UIP), with two non-specific interstitial pneumonia cases, and one with end-stage diffuse alveolar damage.

In the non-sarcoid fibrotic lung disease cohort, there were 40 men and 17 women, with a mean age at transplant of 60 years. The sarcoidosis cohort had three men and five women, and a mean age at transplant of 57 years. The emphysema cohort had nine men and 12 women and a mean age at transplant of 62 years (Table 1).

Over one-third (35\%) of all explant cases had NECH. In the non-sarcoid fibrotic lung disease group, there were three cases of DPNECH (5\%), as well as 20 cases of NECH (35\%), and no cases of borderline DPNECH. The emphysema group had one case of DPNECH (5\%), two cases of borderline DPNECH (10\%), and seven cases with NECH (33\%). The sarcoidosis group had two cases of DPNECH (25\%) and three cases with NECH (38\%), and no cases of borderline DPNECH (Table 2).

One UIP case with DPNECH consisted of a total of 26 tumourlets and 50 bronchioles with NECH (Fig. 1). No significant differences were present among the disease groups, although DPNECH was seen only in the UIP cases of the non-sarcoid fibrotic lung disease cohort. There were no cases of DPNECH in the non-UIP, non-sarcoid fibrotic lung disease cases. No cases had any evidence of obliterative bronchiolitis.

\section{DISCUSSION}

Since being described in the first case series by Aguayo et al. in 1992, ${ }^{9}$ interest and awareness of DIPNECH and the spectrum of neuroendocrine cell disease has increased. There is evidence to suggest that DIPNECH may be under recognised, with more cases possibly coming to light in present day due to increased use of high resolution imaging and biopsy.

Marchevsky et al. recently proposed using at least five pulmonary neuroendocrine cells in a minimum of three separate bronchioles associated with three or more carcinoid tumourlets as the minimal criteria for a histological diagnosis of DIPNECH. In their study, they evaluated consecutive lung biopsy and resection specimens diagnosed with multifocal neuroendocrine cell proliferations rather than consecutive lung specimens resected for any reason. They reported 70 $(9.7 \%)$ cases were diagnosed with multifocal neuroendocrine cell proliferation out of 720 lung specimens with carcinoid

Table 1 Sex distribution and mean age for patients by underlying disease

\begin{tabular}{lll}
\hline Disease process & Male:Female & Mean age \pm SD \\
\hline Fibrotic lung disease (excluding sarcoid) & $40: 17$ & $60 \pm 12$ \\
Emphysema & $9: 12$ & $62 \pm 6$ \\
Sarcoidosis & $3: 5$ & $57 \pm 7$ \\
\hline
\end{tabular}

tumours or tumourlets. Of those 70 cases, 30 (43\%) demonstrated DIPNECH by their criteria. ${ }^{8}$

The results of our study would suggest that $\mathrm{NECH}$ is common in fibrotic lung disease and emphysema. The incidence of NECH has been reported to be as high as $76 \%$ in patients undergoing resection for carcinoid tumours, though the results may have been biased due to the association between NECH and neuroendocrine tumours. ${ }^{4}$ It is possible that the relatively high percentage of cases classified as $\mathrm{NECH}$ in our study is due to the proposed criteria being too inclusive, and further studies of lung specimens with interstitial lung disease and emphysema are needed to verify the frequency of neuroendocrine cell proliferations.

Of note, we did not identify the histological presence of obliterative bronchiolitis, which also was not included as part of the diagnostic criteria proposed by Marchevsky. Obliterative bronchiolitis has been described as a characteristic finding of DIPNECH in several reports, including the first case series by Aguayo et al. ${ }^{9}$ However, only approximately $30 \%$ of patients with pulmonary function test data have shown obstructive changes, and none of the cases in the study by Marchevsky et al. exhibited obliterative bronchiolitis. ${ }^{8,11}$ Furthermore, in our study of lung cases with known interstitial disease, the finding of obliterative bronchiolitis would not be unexpected.

There have been limited studies examining the frequency of normal pulmonary neuroendocrine cells and pulmonary neuroendocrine cell proliferations in normal lung. Weichselbaum et al. demonstrated densities of 65-250 cells per $\mathrm{mm}^{2}$ on individual whole mounts of bronchial epithelium. ${ }^{12}$ A study by Gosney et al. reported approximately six neuroendocrine cells per $10 \mathrm{~cm}$ of airway epithelial length by neurone specific enolase immunoreactivity in autopsy lung specimens. ${ }^{13}$ Mean density of pulmonary neuroendocrine cells for individual bronchi have remained difficult to characterise due to the heterogeneic distribution of the solitary cells. Difficulty in obtaining biopsy or resection tissue from healthy, adult, non-smoker lungs poses a problem as well.

To our knowledge, this is the first series utilising neuroendocrine immunostains to determine the frequency of pulmonary neuroendocrine cell proliferations in lung explants as a primary or secondary finding. Descriptions of DIPNECH in the context of lung transplantation have been limited to a few case reports as a potential treatment in patients with deteriorating pulmonary function. ${ }^{14,15}$

$\mathrm{NECH}$ arising in interstitial lung disease has primarily been reported in the paediatric population, and is part of the diagnostic criteria for neuroendocrine cell hyperplasia of infancy (NECHI). ${ }^{16-21}$ In adults, a study by Wilson et al. compared lungs with interstitial pneumonitis at necropsy, approximately half of which demonstrated frank honeycombing, to controls without pulmonary disease. Pulmonary neuroendocrine cells were found to be decreased in early fibrosis, and absent in areas of honeycombing, despite the fact that all cases contained numerous strips of well preserved epithelium. $^{22}$ A study by Gubbelmans et al. found no difference in the number of neuroendocrine cells per $10 \mathrm{~cm}$ of epithelium between control and anthrasilicotic lungs by chromogranin A immunoreactivity. ${ }^{23}$ A similar study by Gosney et al. also found no significant difference by both protein gene produce 9.5 and chromogranin A, though $10 \%$ less cells were identified by chromogranin A staining. The 
Table 2 Neuroendocrine proliferation in lung transplant recipients based on underlying disease

\begin{tabular}{lllll}
\hline Disease process & $n$ & $n$ with NECH & $n$ with borderline DPNECH & $n$ with DPNECH \\
\hline Fibrotic lung disease (excluding sarcoid) & 57 & $20(35 \%)$ & $0(0 \%)$ & $3(5 \%)$ \\
Emphysema & 21 & $7(33 \%)$ & $2(10 \%)$ & $1(5 \%)$ \\
Sarcoidosis & 8 & $3(38 \%)$ & $0(0 \%)$ & $2(25 \%)$ \\
Totals & 86 & $30(35 \%)$ & $2(2 \%)$ & $6(7 \%)$ \\
\hline
\end{tabular}

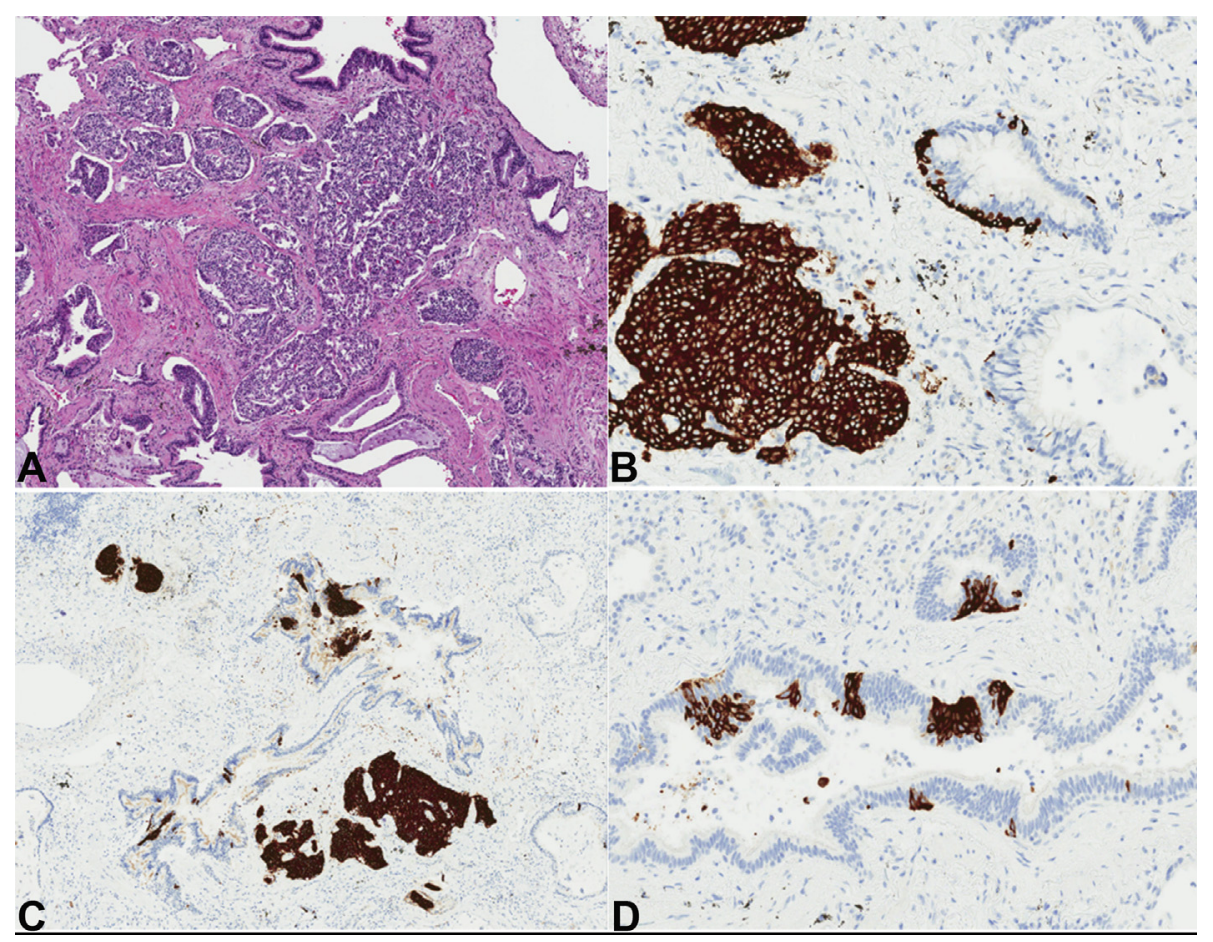

Fig. 1 Index case of DPNECH in a patient with idiopathic pulmonary fibrosis. (A) A carcinoid tumour was identified on H\&E and (B) found to have NECH in an adjacent bronchiole highlighted by synaptophysin staining. (C) Additional tumourlets and (D) bronchioles with NECH were also seen demonstrating synaptophysin positivity.

increase in neuroendocrine cells was also confined to areas of lung affected by acute bronchitis and bronchopneumonia, leading the authors to speculate that inflammation rather than fibrosis is a causative factor of NECH. ${ }^{24}$

Aguayo et al. reported a ten-fold increase in neuroendocrine cells in eosinophilic granuloma by bombesin-like peptide immunoreactivity compared to smokers and patients with idiopathic pulmonary fibrosis. Normal lung tissue was not examined in the study, however, the frequency of approximately 6-8 cells per $10 \mathrm{~cm}$ of airway epithelial length is comparable to the frequency of neuroendocrine cells in normal autopsy lungs as reported by Gosney et al. ${ }^{13,25} \mathrm{~A}$ single case report has described $\mathrm{NECH}$ as a rare cause of interstitial lung disease in two patients, both of whom were female non-smokers living above sea level. Radiological features found on imaging, specifically diffuse ground glass infiltrates in a mosaic pattern, were consistent with NSIP or early hypersensitivity pneumonitis. Of note, these were the only cases diagnosed with $\mathrm{NECH}$ out of 500 consecutive diffuse interstitial lung disease cases studied. ${ }^{26}$ Another case report described DIPNECH diagnosed in a patient with UIP and obliterative bronchiolitis, and other literature have reported UIP-like findings on imaging in association with DIPNECH. $6,27,28$
Literature on NECH as a primary finding in the setting of emphysema is also limited. An early study by Gosney et al. reported increased populations of pulmonary neuroendocrine cells in the lungs of patients with chronic bronchitis or emphysema compared to controls. ${ }^{29}$ A case of DIPNECH arising in a patient with known chronic obstructive disease has also been reported.

A series of case reports identified three patients with bronchial carcinoids and sarcoidosis. ${ }^{30} \mathrm{NECH}$ as a primary finding in association with sarcoidosis otherwise has not been studied.

Overall, NECH is generally a well-accepted process in chronic lung disease, however the literature has been limited mostly to case reports, and there are few studies regarding its incidence and quantification. By utilising the proposed criteria for DIPNECH, this is the first study to quantify NECH by the density of neuroendocrine cells within individual bronchi. The association between $\mathrm{NECH}$ and chronic lung disease raises the possibility that carcinoid tumours and neuroendocrine carcinomas would have an increased incidence in patients with fibrotic lung disease and emphysema. A series of case reports identified three patients with bronchial carcinoids and sarcoidosis. ${ }^{30}$ The series of carcinomas reported by Aubry et al. in patients with idiopathic pulmonary fibrosis included 
squamous cell carcinomas, adenocarcinomas, and a single spindle cell carcinoma, but no case of neuroendocrine tumour. ${ }^{31}$ We know of no association between idiopathic pulmonary fibrosis and carcinoid tumours or neuroendocrine carcinomas, suggesting that the presence of NECH in and of itself is not a high risk for the development of invasive tumours. Similarly, we know of no association between emphysema and neuroendocrine tumours, independent of smoking.

Our results suggest that fibrotic lung disease is a predisposing factor for neuroendocrine cell proliferation, in addition to the known risk of carcinomas. This is consistent with $\mathrm{NECH}$ being recognised as a reactive response to chronic hypoxia, such as in patients living at high altitudes or with a history of cigarette smoking. ${ }^{32}$ Although not addressed in the current study, it has also been identified in patients with a variety of lung diseases, including bronchopulmonary dysplasia, cystic fibrosis, chronic obstructive pulmonary disease, and eosinophilic granuloma. ${ }^{10}$ This is speculated to be due to the fact that PNCs secrete a variety of cytokines and growth factors which can stimulate fibroblasts and airway cell chemotaxis, resulting in bronchiolar fibrosis. ${ }^{33}$

Currently, the distinction between DIPNECH and a reactive neuroendocrine cell proliferation is based on histopathological criteria as well as clinical context, and it is unclear whether there is an actual difference in the pulmonary neuroendocrine cells proliferating in DIPNECH versus those reacting to lung injury. More recent studies have identified a different immunohistochemical profile in DIPNECH compared to reactive $\mathrm{NECH}$, specifically a more consistent and earlier expression of cell-cycle proteins p53, Ki-67, and p16 in DIPNECH. Neuroendocrine cell proliferations in DIPNECH are also believed to be more florid than in reactive $\mathrm{NECH}$, so applying the diagnostic criteria for DIPNECH may actually be too restrictive in identifying neuroendocrine cell proliferations in response to lung injury. ${ }^{34,35}$

Conflicts of interest and sources of funding: The authors state that there are no conflicts of interest to disclose.

Address for correspondence: Susan Shyu, MD, Department of Pathology, University of Maryland Medical System, 22 S. Greene St, NWB43, Baltimore, MD, 21201, USA. E-mail: susanshyu@gmail.com

\section{References}

1. Hendifar AE, Marchevsky AM, Tuli R. Neuroendocrine tumors of the lung: current challenges and advances in the diagnosis and management of well-differentiated disease. J Thorac Oncol 2017; 12: 425-36.

2. Travis WD, Brambilla E, Burke AP, et al., editors. WHO Classification of Tumours of the Lung, Pleura, Thymus, and Heart. 4th ed. Lyon: IARC, 2015; 78-9.

3. Miller MA, Mark GJ, Kanarek D. Multiple peripheral pulmonary carcinoids and tumorlets of carcinoid type, with restrictive and obstructive lung disease. Am J Med 1978; 65: 373-8.

4. Miller RR, Muller NL. Neuroendocrine cell hyperplasia and obliterative bronchiolitis in patients with peripheral carcinoid tumors. Am J Surg Pathol 1995; 19: 653-8.

5. Dahiya M, Husain AN. Pathologic quiz case: patient with chronic obstructive pulmonary disease. Diffuse pulmonary neuroendocrine hyperplasia with multifocal tumorlets, immunoreactive to synaptophysin, associated with pulmonary emphysema. Arch Pathol Lab Med 2000; 124: $171-3$.

6. Pietrangeli V, Piciucchi S, Tomassetti S, et al. Diffuse neuroendocrine hyperplasia with obliterative bronchiolitis and usual interstitial pneumonia: an unusual "headcheese pattern" with nodules. Lung 2015; 193 : $1051-4$.
7. Aubry MC, Thomas Jr CF, Jett JR, et al. Significance of multiple carcinoid tumors and tumorlets in surgical lung specimens: analysis of 28 patients. Chest 2007; 131: 1635-43.

8. Marchevsky AM, Wirtschafter E, Walts AE. The spectrum of changes in adults with multifocal pulmonary neuroendocrine proliferations: what is the minimum set of pathologic criteria to diagnose DIPNECH? Hum Pathol 2015; 46: 176-81.

9. Aguayo SM, Miller YE, Waldron Jr JA, et al. Brief report: idiopathic diffuse hyperplasia of pulmonary neuroendocrine cells and airways disease. N Engl J Med 1992; 327: 1285-8.

10. Davies SJ, Gosney JR, Hansell DM, et al. Diffuse idiopathic pulmonary neuroendocrine cell hyperplasia: an under-recognised spectrum of disease. Thorax 2007; 62: 248-52.

11. Marchevsky AM, Walts AE. Diffuse idiopathic pulmonary neuroendocrine cell hyperplasia (DIPNECH). Semin Diagn Pathol 2015; 32 $438-44$.

12. Weichselbaum M, Sparrow MP, Hamilton EJ, et al. A confocal microscopic study of solitary pulmonary neuroendocrine cells in human airway epithelium. Respir Res 2005; 6: 115.

13. Gosney JR, Sissons MC, Allibone RO. Neuroendocrine cell populations in normal human lungs: a quantitative study. Thorax 1988; 43: 878-82.

14. Zhou H, Ge Y, Janssen B, et al. Double lung transplantation for diffuse idiopathic pulmonary neuroendocrine cell hyperplasia. J Bronchol Interv Pulmonol 2014; 21: 342-5.

15. Sheerin N, Harrison NK, Sheppard MN, et al. Obliterative bronchiolitis caused by multiple tumourlets and microcarcinoids successfully treated by single lung transplantation. Thorax 1995; 50: 207-9.

16. Cazzato S, di Palmo E, Ragazzo V, et al. Interstitial lung disease in children. Early Hum Dev 2013; 89 (Suppl. 3): S39-43.

17. Das S, Langston C, Fan LL. Interstitial lung disease in children. Curr Opin Pediatr 2011; 23: 325-31.

18. Caimmi S, Licari A, Caimmi D, et al. Neuroendocrine cell hyperplasia of infancy: an unusual cause of hypoxemia in children. Ital J Pediatr 2016; 42: 84 .

19. Young LR, Brody AS, Inge TH, et al. Neuroendocrine cell distribution and frequency distinguish neuroendocrine cell hyperplasia of infancy from other pulmonary disorders. Chest 2011; 139: 1060-71.

20. Yancheva S, Velani A, Rice A, et al. Bombesin staining in neuroendocrine cell hyperplasia of infancy (NEHI) and other childhood interstitial lung diseases (chiLD). Histopathology 2015; 67: 501-8.

21. Cutz E, Yeger H, Pan J. Pulmonary neuroendocrine cell system in pediatric lung disease-recent advances. Pediatr Dev Pathol 2007; 10: 419-35.

22. Wilson NJ, Gosney JR, Mayall F. Endocrine cells in diffuse pulmonary fibrosis. Thorax 1993; 48: 1252-6.

23. Gubbelmans HL, Maesen BL, Koudstaal J, et al. Neuroendocrine cells in anthracosilicotic lungs. Eur Respir J 1991; 4: 824-8.

24. Gosney JR, Peers J, Beesley C, et al. Pulmonary endocrine cells in anthracosilicotic lungs. Eur Respir J 1997; 10: 388-91.

25. Aguayo SM, King Jr TE, Waldron Jr JA, et al. Increased pulmonary neuroendocrine cells with bombesin-like immunoreactivity in adult patients with eosinophilic granuloma. J Clin Invest 1990; 86: 838-44.

26. Reyes LJ, Majó J, Perich D, et al. Neuroendocrine cell hyperplasia as an unusual form of interstitial lung disease. Respir Med 2007; 101: 1840-3.

27. Chatterjee K, Kamimoto JJ, Dunn A, et al. A case of DIPNECH presenting as usual interstitial pneumonia. Pneumonol Alergol Pol 2016; 84: $174-7$.

28. Lyall MS, Evens S, Remmen H, et al. Diffuse idiopathic pulmonary neuroendocrine cell hyperplasia: a case associated with UIP-like fibrosis J Pathol 2005; 207: 13S.

29. Gosney JR, Sissons MC, Allibone RO, et al. Pulmonary endocrine cells in chronic bronchitis and emphysema. J Pathol 1989; 157: 127-33.

30. Levy NT, Rubin J, DeRemee RA, et al. Carcinoid tumors and sarcoidosis-does a link exist? Mayo Clin Proc 1997; 72: 112-6.

31. Aubry MC, Myers JL, Douglas WW, et al. Primary pulmonary carcinoma in patients with idiopathic pulmonary fibrosis. Mayo Clin Proc 2002; 77: 763-70

32. Gorshtein A, Gross DJ, Barak D, et al. Diffuse idiopathic pulmonary neuroendocrine cell hyperplasia and the associated lung neuroendocrine tumors: clinical experience with a rare entity. Cancer 2012; 118: 612-9.

33. Baniak NM, Wilde B, Kanthan R. Diffuse idiopathic pulmonary neuroendocrine cell hyperplasia (DIPNECH) - An uncommon precursor of a common cancer? Pathol Res Pract 2016; 212: 125-9.

34. Gosney JR, Williams IJ, Dodson AR, et al. Morphology and antigen expression profile of pulmonary neuroendocrine cells in reactive proliferations and diffuse idiopathic pulmonary neuroendocrine cell hyperplasia (DIPNECH). Histopathology 2011; 59: 751-62.

35. Chassagnon G, Favelle O, Marchand-Adam S, et al. DIPNECH: when to suggest this diagnosis on CT. Clin Radiol 2015; 70: 317-25. 